\title{
Autour du hasard et dans le sol
}

\section{BOISSIER}

C. BACCONNET

LGC/CUST -

Université Blaise-Pascal

BP 206

63174 Aubière

d.boissier@cust.univbpclermont.fr

c.bacconnnet@cust.univbpclermont.fr

J. ALHAJJAR

Laboratoire de génie civil IUT de Saida

Université libanaise de Beyrouth BP 813

Saida, Liban

j.hajjar@iutsaida.edu.li
Cet article présente une réflexion sur la signification de ce que l'on met souvent sous les termes « hasard $»$ ou « incertain » pour un chercheur mécanicien des sols et pour un praticien géotechnicien. Par des exemples relatifs à des études réalisées (ouvrages, sols urbains), cet article montre ce que peuvent apporter les différentes techniques issues des statistiques et de la théorie de l'information.

Mots-clés : analyse de risque, fiabilité, méthodes statistiques et probabilités, ensembles flous.

\section{About random and within soil}

practice in geotechnics are concerned. From examples based on developed studies, (dams, dikes, snow slopes, networks, buildings, urban soils), this paper shows the interest of methods based on statistics and information theory approaches.

Key words: risk analysis, reliability, statistics and probabilities, fuzzy sets. 


\section{Introduction : l'ingénieur et le sol}

Les sols sont tous différents de par leur morphologie, leur comportement, leurs réactions à des agents pathogènes, à des sollicitations, à l'effet du temps...

Chaque sol est unique et il est, en plus, différent selon l'environnement dans lequel il se situe, différent d'un moment à l'autre, d'une ère à l'autre. Le domaine du sol « géo ») est fait de cas particuliers; or, il n'y a de science que du général ; alors comment peut-il y avoir une science du sol ? C'est en particulier pour répondre à cette question que les méthodes issues de la statistique et leurs dérivées ont été utilisées.

Dans le domaine des sciences pour l'ingénieur concerné par le sol urbain, trois difficultés sont centrales : une difficulté d'ordre social qui est celle de la prise de risque et de la décision, une difficulté d'ordre technique et technologique qui est celle de J'observation et de la mesure et une difficulté d'ordre scientifique qui est celle de la connaissance des phénomènes et des mécanismes. Or, dans le domaine du sol urbain ces trois problèmes, peut-être encore d'avantage que dans le domaine du génie civil traditionnel, se posent dans un contexte difficile où incertain et imprécis cohabitent. Nous voulons préciser ici ce que signifie le ( hasard » et ce que peuvent apporter les outils et méthodes le prenant en compte pour chacune de ces préoccupations.

\section{2}

\section{Domaines de connaissance et sols}

\section{1}

\section{Connaissance, non-connaissance, aléa, incomplétude}

Il est trivial d'énoncer que l'ingénieur ne connaît pas tout ; il est utile de différencier son domaine d'incapacité - celui de sa non-connaissance -, de son domaine de compétence - celui de sa connaissance. Nous nous plaçons ici dans le domaine de la connaissance seule, en observant que, même dans ce domaine, tout n'est pas parfait, la compétence de l'ingénieur étant souvent limitée à cause des imprécisions, de l'aléa du futur, de la non-formalisation des connaissances, de l'apparente contradiction des mesures ou des déclarations.

\section{2ir.t}

\section{Connaissance et non-connaissance}

Le premier volet du domaine de non-connaissance est celui où l'on ne connaît pas l'existence d'objets ou d'événements passés et où il n'est donc pas possible d'en analyser les conséquences éventuelles ; par exemple, relève de ce type de non-connaissance l'existence de cavités, d'ouvrages ou de sols anthropiques dans des zones où ni la mémoire collective, ni des travaux d'archéologie n'ont conservé d'informations. Un autre volet de cette notion de non-connaissance est celle de la non-connaissance de phénomènes physiques : il y a cent ans, par exemple, les phénomènes d'alcali-réaction n'étaient pas connus, et il n'était pas possible alors d'en prévoir les conséquences néfastes sur les ouvrages et, encore moins, de tenter de s'en prémunir en formulant différemment les bétons ; l'histoire des sciences montre que chaque époque découvre des problèmes dont les générations antérieures n'avaient pas eu connaissance.

L'activité de l'ingénieur ne peut se situer qu'à l'intérieur d'un domaine de connaissances, et la notion de prise de risque et de décision n'est significative que dans ce contexte ; c'est dans ce contexte de connaissance que nous développons la suite de ce texte.

\section{1.?}

\section{Aléa et déterminisme}

Nous nous situons maintenant dans le domaine de la connaissance qui est celui où les événements, ayant pu se produire dans le passé ou pouvant se produire dans le futur, sont identifiés : actions et sollicitations, changements d'état des systèmes, mécanismes de dégradation... Dans ce domaine, deux sous-domaines peuvent être définis : celui du déterminisme et celui de l'aléa.

Le domaine du déterminisme est celui où l'ingénieur contrôle toutes les informations (ou les contrôle suffisamment pour que le rôle des informations non contrôlées soit négligeable) ; c'est celui de la transmission traditionnelle du savoir académique où nulle incertitude n'entachant ni les données, ni les hypothèses, ni les modèles, l'étudiant pense que les résultats sont certains! Ce n'est évidemment pas ce domaine qui nous préoccupe ici.

Le domaine de l'aléa peut être divisé en deux domaines très importants tous deux en ce qui concerne le sol urbain : celui de a l'aléa du passé n et celui de « l'aléa du futur ». Le domaine de " l'aléa du passé \# est celui d'une épreuve qui a eu lieu, l'aléa résidant dans le fait que nous ne connaissons pas de manière certaine quels ont été les résultats (modalités présentes) de l'épreuve et que nous n'avons que des présomptions, des éléments de preuve. Nous pouvons alors associer à chacune des modalités, dont la réalisation était possible à l'issue de cette épreuve, une mesure de la croyance que nous avons de sa réalisation. Cette démarche, classique chez les historiens, l'est moins chez les ingénieurs qui se contentent trop souvent, en logique binaire, de la certitude ou de l'impossibilité d'apparition d'une modalité. A l'opposé, l'aléa du futur est celui d'une épreuve aléatoire qui n'a pas encore eu lieu et qui n'aura d'ailleurs peut-être pas lieu ; dans le cas de sa réalisation, on ignore, bien sûr, quel en sera le résultat. Deux types de problèmes peuvent alors se présenter : soit l'épreuve ne ressemble à aucune autre épreuve connue déjà réalisée, on ne dispose alors d'aucune indication sur les modalités possibles ni sur leur probabilité d'apparition, et on se retrouve dans un problème d'inconnaissance ; soit l'épreuve peut être considérée comme une des épreuves élémentaires d'une épreuve composée ou comme la conséquence d'une épreuve connue, alors l'observation des modalités déjà réalisées, de leur fréquence d'apparition permet de se placer dans le domaine du calcul des probabilités. 


\section{9 .3}

\section{Quantitatif et qualitatif}

L'analyse du passé, support de la prévision, est parfois organisable sur des données quantitatives ; c'est alors le terrain favori d'expression de l'ingénieur et de nombreux outils existent. Parfois aussi, ces données sont, par nature ou par facilité, exprimées sous forme qualitative, à l'issue d'observations ou de déclarations d'experts; c'est là aussi un langage d'ingénieur que les facilités et la suprématie du calcul ont, pendant une période, réduit jusqu'à l'apparition et le développement de formulations basées sur le qualitatif et sur la logique floue en particulier.

\section{4}

\section{Le concordant et le discordant}

Quelles soient numériques ou qualitatives, issues de mesures ou d'observations, les informations ne sont pas toujours concordantes. Qui n'a un jour éliminé de façon arbitraire un point « aberrant » d'une courbe, omis un avis discordant dans une expertise? Pourtant, toute information doit être analysée au double regard de son informativité - l'intérêt de ce quelle apporte - et de sa fiabilité. Là aussi, il y a nécessité de méthodes permettant d'unifier des informations en tenant compte de la qualité de leur source.

\section{2}

\section{Le cas des sols et des ourrages}

Dans le cas des sols, ces divers domaines de la connaissance de l'aléa, ces différentes formes de l'information cohabitent et, par conséquence, on les retrouve sur la table de travail de l'ingénieur. Dans ce paragraphe nous illustrons ces notions.

\section{9}

\section{L'univers de non connaissance des objets et des problèmes}

A un instant donné, cet univers est celui des travaux qui concerneront dans trente, cent ou mille ans la zone concernée ; c'est dans ce contexte que se situe le problème du stockage des déchets radioactifs lié à la perte de mémoire par les générations futures de la localisation des stockages ou, même, des dangers que présentent ces stockages ; c'est celui aussi, de la découverte fortuite d'un branchement de réseau que l'on avait oublié, d'un tronçon qui desservait une zone maintenant friche industrielle...

\section{$292-2$}

\section{L'univers " aléa du passé »}

Quelques exemples illustrent ce phénomène : en géologie, en un endroit donné, la couche d'argile incluse dans deux couches perméables existe ou n'existe pas et est le résultat éventuel d'une épreuve géologique très ancienne. Sa présence n'est donc pas aléatoire, c'est la connaissance de cette présence (ou de son absence ) qui est déficiente. De même, dans un sous-sol urbain, dans une zone donnée, on peut savoir qu'il y a eu une activité humaine d'extraction de matériaux, par contre, il est plus difficile de localiser précisément cette activité et les carrières souterraines qui en ont résulté.$$
\text { 2..2.3.3 }
$$

\section{L'univers d'incomplétude de la connaissance des objets du sol}

C'est celui de la mauvaise connaissance de l'histoire des ouvrages : conditions de réalisation, histoire de sollicitations, d'entretien, de pathologie, de réparation. L'incomplétude provient aussi de la faiblesse et de la disparité de contenu des bases de données urbaines. Enfin, plus fondamentalement, les réseaux et les ouvrages dans les sols urbains sont enterrés et cachés et, de même que pour le sol environnant, il est impossible d'en avoir une connaissance exhaustive.

\section{2.,25:}

\section{L'univers aléatoire de l'avenir des ouvrages}

L'aléa est ici celui lié au développement de la ville, de ses besoins en eau et en assainissement, de l'imperméabilisation en surface, des projets d'infrastructures, C'est aussi celui lié aux sollicitations hydrauliques s'exerçant sur les réseaux et causées par les intensités et les fréquences des orages, aux quantités et qualités des eaux de ruissellement. Enfin c'est l'aléa des sollicitations mécaniques exercées sur les conduites par les charges en surface, les interactions entre ouvrages et les mouvements des sols.

\section{2 .5}

\section{L'univers déterministe des actions humaines sur les ouvrages}

C'est celui des actions humaines volontaires affectant la production d'un ouvrage : obturation d'un tronçon, branchement sauvage, rejet volontaire de polluants. C'est aussi celui des erreurs techniques (mauvaise appréciation de l'état d'une conduite, campagne de reconnaissance insuffisante, mauvais choix de technique de confortation d'un ouvrage). C'est, enfin, celui de la faute du gestionnaire ou de l'élu, ignorant volontairement un problème, ou de l'entreprise ne respectant pas une qualité de compactage ou de matériau.$$
\text { 2.2.6 }
$$

\section{L'univers aléatoire du vieillissement des ouvrages}

Le vieillissement des ouvrages est un phénomène normal dont l'évolution est aléatoire et qui est de plus en plus pris en compte par les ingénieurs ; la courbe " en baignoire » caractéristique des trois périodes de la vie des équipements et des ouvrages - les dégradations de jeune âge, l'époque de fonctionnement normal, puis celle de l'accélération des dégradations - est bien connue, mais ses paramètres sont aléatoires.

Les ouvrages du sol urbain relèvent de cette problématique. On peut noter, pour les ouvrages récents, une diminution de l'échelle des temps (espérance de la durée de vie d'une canalisation romaine bien plus grande que celle d'une canalisation du $\mathrm{XX}^{e}$ siècle). 


\subsection{7.}

\section{L'univers aléatoire des sollicitations extrêmes}

Les ouvrages dans les sols urbains sont parfois soumis à des combinaisons d'actions exceptionnelles. Chaque sollicitation relève d'un phénomène, dont la probabilité d'occurrence des valeurs extrêmes peut être estimée. La difficulté provient de la simultanéité ou de la concomitance de plusieurs phénomènes. Ainsi, par exemple, la liquéfaction de sols est liée à la concomitance de la saturation d'un sol fin et de l'occurrence d'une forte sollicitation sismique.

Les catastrophes liées à des inondations sont souvent la conjonction d'un phénomène climatique exceptionnel et d'une succession de fautes et d'erreurs humaines, de vieillissement. Les inondations de Nimes sont dues à un épisode de pluies torrentielles exceptionnelles et à l'accumulation de mauvaises décisions techniques, comme la couverture des cours de petits ruisseaux appelés « cadereaux 》, l'imperméabilisation des sols et des rues, le mauvais entretien et la vétusté des réseaux.

\section{3}

\section{Prise de risque, décision et responsabilité}

\section{1}

\section{Définitions}

\section{1}

\section{Risque, défaillance, danger et dommage}

Le problème du risque, dans le contexte du génie civil et urbain, se pose en terme d'acceptabilité d'un certain niveau de risque. Ce problème fait référence à d'autres notions, telles que le danger, les accidents, les défaillances et les dommages, termes que nous devons définir.

Selon la norme NF F 71-011, le risque est la probabilité d'occurrence d'une défaillance associée à l'importance des conséquences de celle-ci.

Au sens du Petit Larousse, le danger est « une situation où l'on a à redouter un inconvénient, ou un mal quelconque $)$.

D'après la norme NF F 71-011, l'accident est « l'événement ou succession d'événements imprévus, ayant pour résultat une atteinte à l'intégrité physique des personnes ou des destructions de matériel ». Un accident est un événement ou une succession d'événements, ce n'est pas un état. Un accident provoque des dommages pour les personnes, le système ou l'environnement.

La notion de défaillance est abordée dans la norme française X 60-500 d'octobre 1988 intitulée « Terminologie relative à la fiabilité, maintenance, disponibilité $x$. Cette norme définit la défaillance comme " la cessation de l'aptitude d'une entité à accomplir une fonction requise $n$.
Le dommage est défini, selon le Petit Larousse, comme une " perte, dégât causé à quelque chose, préjudice causé à quelqu'un ».

\section{2}

\section{Méthodes d'analyse du risque et d'aide à la décision}

L'analyse du risque peut être traitée qualitativement et quantitativement.

Qualitativement parlant, lorsqu'il y a une source de danger et lorsqu'il n'y a pas de moyens de sécurité contre l'exposition au danger, alors il y a une possibilité de défaillance ou d'accident. Cette possibilité est assimilée à un risque, la défaillance ou l'accident pouvant venir des activités de commerce, sociales ou militaires, des opérations d'équipement ou d'investissement. Le risque peut être, alors, formellement défini comme la possibilité d'une défaillance ou d'un accident résultant d'une exposition à un danger. Bien que l'analyse quantitative du risque repose sur l'estimation du degré ou de la probabilité de défaillance, elle est fondamentalement mêlée au concept de probabilité d'occurrence du danger et consiste à répondre aux questions suivantes :

a) Que peut-il arriver qui peut conduire au résultat d'une exposition au danger?

Pour répondre à la première question, une liste des défaillances possibles doit être définie (ou scénario d'événements conduisant à une défaillance).

b) Est-ce que cela va arriver?

La probabilité d'occurrence de ces scénarios doit être définie et évaluée, et on peut, alors, classer les scénarios par leur probabilité d'occurrence.

c) Si cela arrive, à quelles conséquences peut-on s'attendre?

On doit associer à chaque scénario les modalités des conséquences (déterministes ou aléatoires) et les évaluer.

De cette manière, le risque peut être défini quantitativement, par le triplet suivant:

$$
R=\langle S i, P i, C i\rangle, i=1,2 \ldots, n
$$

où : Si est un scénario d'événements qui conduisent à une exposition à un danger :

Pi est la probabilité du scénario $i$ (ou la possibilité, ou...) ;

Ci est la conséquence (ou le résultat d'une évaluation) du scénario $i$, c'est-à-dire une estimation du degré de dommage ou de défaillance.

Les niveaux de risque, selon la norme NF F 00-101, sont représentés sous forme d'une matrice gravité/occurrence. La norme NF F 00-101 ne définit qu'un niveau de risque acceptable, auquel il est naturel d'ajouter le niveau complémentaire. Les deux niveaux de risque sont donc les risques inacceptables et, implicitement, les risques acceptables. Chaque point sensible est analysé en fonction de trois facteurs : probabilité d'apparition des défaillances, gravité des défaillances, incertitude de détection des défaillances. 


\section{3}

\section{Respect de la réglementation et responsabilité}

La notion de risque est au cœur de l'activité de l'ingénieur à travers les aspects respect de la réglementation et mise en cause de sa responsabilité que comporte sa profession. Les professionnels engagent leur responsabilité en cas de désordre survenant aux constructions; le problème de fond n'est pas seulement celui de la prévision de phénomènes aléatoires ou non, mais plutôt celui de l'acquisition d'informations existantes, mais peu ou pas accessibles. En fait, se prémunit-on d'un événement exceptionnel qui se produit ? L'ingénieur est-il responsable de ne pas avoir dimensionné sa structure pour résister à un séisme de magnitude 8 ou d'avoir surestimé la résistance du sol ?

\section{4}

\section{La connaissance des phénomènes et la prévision des comportements}

L'activité de l'ingénieur se complique de la nécessité de modéliser une réalité compliquée par des modèles qui, même complexes, restent des modèles donc des idéalisations.

\section{Les modèles}

Le comportement d'un ouvrage dans un sol dépend de sa géométrie, des conditions aux limites et des matériaux constitutifs; la prévision de ce comportement (ouvrage neuf ou ouvrage en service) dépend des objectifs qui lui sont assignés : court ou long terme, petites déformations ou rupture, sollicitation statique ou dynamique ou de fatigue, et se base sur des modèles de comportement qui sont liés aux modèles de sol ; on ne peut découpler modèle de comportement et modèle de sol ; or, le modèle de sol dépend essentiellement des données disponibles.

Ces modèles sont orientés vers deux directions. En application tout d'abord : leur objectif est d'aider l'ingénieur à vérifier que le comportement " possible » ou " probable » est compatible avec l'objectif poursuivi et à argumenter alors la prise de risque. En recherche ensuite : leur objectif est de mieux identifier les mécanismes conduisant à tel comportement d'ouvrage ; les modèles servent à augmenter le domaine de connaissance et à diminuer d'autant celui de la non-connaissance. Durant les dernières décennies, on a créé des modèles de sol et de comportement des ouvrages de plus en plus sophistiqués en négligeant la part de l'acquisition et du traitement des données.

Les modèles sont, eux aussi, facteurs d'incertitude. Tout modèle n'est ni vrai ni faux, il n'est acceptable que dans un cadre d'hypothèses donné et pour un objectif fixé. Les incertitudes des modèles proviennent en premier facteur de l'écart entre le cadre d'hypothèse sur lesquelles ils ont été bâtis et la réalité des phénomènes en jeu. Le deuxième facteur est lié à la manière dont les modèles transforment les incertitudes sur les entrées ; l'axiome « mauvaises données, mauvais résultats » doit être tempéré par des analyses de sensibilité des résul- tats aux incertitudes sur les données. C'est une voie pour mieux cerner quelles sont les données dont il faut diminuer le domaine d'imprécision, afin, dans le cas d'une application, d'optimiser les campagnes de reconnaissance des sols et, dans le cas d'une recherche, de construire les plans d'expérience les plus efficaces.

\subsection{9}

\section{Prise en compte de la variabilité}

La mécanique des sols est une perpétuelle recherche du volume élémentaire représentatif (VER) du problème que l'on veut traiter. On peut se placer aux différentes échelles de la mécanique atomique, de la mécanique des milieux granulaires ou de la mécanique des milieux continus, le VER étant celui dans lequel un volume de matériau peut être considéré comme homogène.

S'il y a homogénéité dans un VER, c'est qu'il y a variabilité d'un VER à l'autre. Alors, la formulation d'un problème passe par la description de la population des VER utilisée pour la caractérisation par une moyenne (par exemple pour la cohésion) ou par un pourcentage (par exemple pour la saturation).

La difficulté est qu'on ne peut jamais accéder à toute la population, c'est-à-dire à tous les VER d'un sol : on ne dispose que d'échantillons, la statistique permettant alors de remonter aux paramètres inconnus « cohésion moyenne » ou a pourcentage moyen de saturation » par la fréquence d'apparition dans la population à travers une fourchette (intervalle de confiance) qui dépend de la taille de l'échantillon. Et c'est là que le bât blesse en ce qui concerne les sols, car l'échantillon comporte toujours peu de valeurs et, le plus fréquemment, une seule, et qu'on ne peut déterminer de fourchette que sí l'échantillon est représentatif de la population, ce qui n'est réalisé que śil est tiré au sort.

Dans cette démarche, on peut, en gros, distinguer deux types de problèmes. Le premier est de type opérationnel : c'est la description d'un sol pour obtenir une caractéristique, un événement, sous une sollicitation donnée, par exemple, la cohésion (variable quantitative) ou le fait d'être ou non saturé (variable qualitative). Le second type de problèmes est de type recherche, c'est la description comparée. On veut savoir s'il existe une liaison (éventuellement causale) entre deux variables, par exemple, entre une résistance mécanique (cohésion) et une pathologie (rupture ou tassement), ou entre une histoire de chargement et une pathologie (liquéfaction, écoulement, cisaillement, rupture.....). Dans ce cas-lă, une méthode possible est la comparaison de moyennes (cohésion) ou de pourcentage (rupture...). La difficulté est encore ici qu'on ne dispose que d'échantillons ne donnant que des estimations des moyennes et de pourcentages, et qu'il faut aussi, ici, juger sur " échantillons ». Le test statistique est une solution permettant de savoir si la différence des effets est imputable aux fluctuations d'échantillonnage ou si elle est significative : si la différence est significative, elle ne traduit pas nécessairement une relation causale, ceci n'étant vrai que si les échantillons sont comparables, ce qui nécessite leur constitution par tirage au sort.

Un apport de la statistique dans ce type de problèmes est une définition de la causalité dans le domaine de l'incertain. Un facteur causal n'entraîne pas nécessairement l'événement, il suffit qu'il entraîne 
une augmentation de la probabilité d'occurrence de cet événement. Cette approche a été longue a émerger, car nous avons tous été habitués et éduqués dans l'univers du certain, et parce que nous avons peur de l'incertain.

\section{4}

\section{Acquisition d'information et modèles de données}

\section{1}

\section{La difficulté d'obtention des mesures}

Quels que soient le domaine de connaissance et le modèle d'aide à la décision choisi, l'acquisition de connaissances sur les sols urbains et les ouvrages est un point de passage forcé. Ce paragraphe décrit rapidement quelques méthodes d'acquisition et les apports des principales méthodes de traitement.

Milieu continu ou granulaire, ou fracturé, le sol n'est, en général, connu que de manière discontinue : récents, les ouvrages sont documentés, anciens ils ne le sont qu'incomplètement et irrégulièrement, et, dans tous les cas, l'information est fragmentée, répartie entre les gestionnaires, codées selon des règles différentes.

\subsection{1.}

\section{Les méthodes géophysiques}

Elles tentent de donner d'un sol ou d'un ouvrage, une image continue. Leur application est souvent limitée par les perturbations des mesures induites par les sites urbains, par la difficulté d'interprétation des signaux et par les connaissances qualitatives qu'elles induisent. Les méthodes issues du traitement du signal, les techniques actuelles de traitement de l'information et, en particulier, les méthodes basées sur le raisonnement qualitatif sont des éléments de réponse à l'analyse des signaux provenant des méthodes géophysiques.

\subsection{2}

\section{Les méthodes de reconnaissance in situ}

Basées sur des techniques de pénétration, perforation ou carottage, et permettant l'identification des sols rencontrés, la mesure de paramètres mécaniques de résistance, de déformations ou de flux, elles apportent des informations ponctuelles ou continues le long d'un sondage. Ces informations sont souvent de qualité, mais, à cause de leur coût d'acquisition, restent peu nombreuses sur une opération.

Les apports actuels des méthodes à base de statistique sur ces essais sont multiples :

- aide au traitement des signaux qu'elles donnent (stabilité, reconnaissance de couches, signification de la dispersion ou des premiers moments);

- aide à l'analyse de la relation entre plusieurs signaux provenant du même appareil ou de deux appareils de principe différent, recalage spatial et temporel.

\subsection{3.}

\section{Les méthodes d'analyse en semi-grandeur sur banc d'essai}

Elles offrent en plus des essais in situ la possibilité de bien contrôler la variabilité des matériaux en place, les sollicitations appliquées, les signaux enregistrés. Les apports actuels des méthodes à base de statistique sur ces essais sont multiples, aussi on peut citer :

- l'aide à l'analyse de la répétatibilité des essais ;

- l'aide au calibrage des appareils de mesure ;

- l'aide à la mise en place de plans d'expérimentation contrôlés dans le contexte d'études de sensibilité, d'analyse de corrélation.

\subsection{8.}

\section{Les caractérisations au laboratoire}

Elles apportent des analyses de comportement de matériaux sous sollicitations complexes bien contrôlées. Les échantillons sont très souvent remaniés, et le problème de la représentativité du volume de sol testé (volume élémentaire représentatif) est prépondérant. Les apports actuels des méthodes, à base de statistique, sur ces essais sont identiques à celles présentées au paragraphe précédent.

\section{2}

\section{Les bases de données}

\subsubsection{1 \\ L'utilisation de bases de données matériaux}

Le comportement d'un sol sous sollicitation mécanique est conditionné par le matériau (composition minérale, granulométrie, forme des grains...), un indicateur de teneur en eau et l'état de serrage des grains (indice de compactage, indice des vides, poids volumique des grains...). Une base de données comportant pour chaque matériau des descriptions de ces trois champs, ainsi que des réponses à diverses sollicitations mécaniques (pénétration, cisaillement, enfoncement...) permettrait non seulement de stocker de l'information provenant d'expérimentations onéreuses, mais, surtout, de déduire des caractéristiques non connues d'un matériau. Là aussi, les outils issus de la taxinomie, la généralisation de la notion de distance entre des individus (ici des matériaux) sont précieux.

\section{4., .9}

\section{L'utilisation de bases de données sols et ouvrages}

Les ouvrages dans les sols urbains ne sont, en général, pas mieux connus que les sols qui les entourent. Or, la connaissance de leur état est nécessaire à qui souhaite mettre en place une stratégie d'inspection, maintenance, réparation (IMR) qui soit optimale ou, tout simplement, moins mauvaise. La reconnaissance exhaustive des ouvrages n'est, en général, pas possible; il faut alors travailler avec de l'information incomplète et souvent erronée, comme 
le montre l'analyse du contenu des bases de données urbaines existantes. Les techniques basées sur la statistique sont aussi précieuses pour caractériser la qualité des informations déduites, pour aider à définir les plans de reconnaissance et d'auscultation : les réseaux de neurones ont aussi été utilisés dans ce contexte.

\section{3}

\section{Le recueil d'informations}

Les informations sur les sols ne sont pas toujours purement quantitatives. Le " bon sol », un " sol compressible », une " portance d'environ 0,2 $\mathrm{MPa}$ », une "couche homogène », une "cohésion entre 20 et $30 \mathrm{kPa}$, un « sol saturé $n$, la « fondation est sur-dimen-

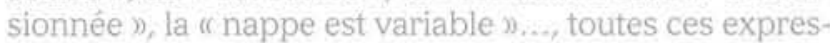
sions ont en commun deux caractéristiques :

1) elles sont communément utilisées et comprises par des géotechriciens ;

2) elles sont toutes à support d'information non précis.

Il y a nécessité d'approches non traditionnelles pour la prise en compte de ce type d'information par les mécaniciens des sols.

Ce type d'information provient souvent de déclarations d'experts. Au fur et à mesure que la complexité des systèmes augmente, notre aptitude à formuler de manière précise et significative leur comportement diminue jusqu'à une limite au-delà de laquelle la précision et la signification deviennent des caractéristiques mutuellement exclusives. Notre connaissance du monde réel est imprécise et celle de son évolution incertaine. Il est vain alors de vouloir la décrire par des modèles précis et déterministes, Cela est vain et, surtout, dangereux, car le sentiment de précision est illusoire, la signification des résultats est très pauvre et les décisions sont prises sur la base d'informations imprécises et sans signification.

Deux arguments sont alors mis en évidence : la fiabilité de l'information et son « informativité »; il apparait vite que ces deux arguments sont souvent conflictuels.

a) Exemple 1. La cohésion du sol dans cette couche est de $20 \mathrm{kPa}$. Cette information est très informative, et l'on peut faire des calculs ou prendre des décisions sur cette base, par contre, elle est très peu fiable. En effet, en pratique, on ne peut pas assurer que le sol a une cohésion de $20 \mathrm{kPa}$, tout au plus peut-on dire que la mesure, dans telle condition, sur tel appareil, de tel échantillon provenant de tel prélèvement, a donné une valeur que l'on arrondit à $20 \mathrm{kPa}$.

b) Exemple 2. La cohésion du sol dans cette couche est comprise entre $10 \mathrm{kPa}$ et $100 \mathrm{kPa}$. Cette information est très fiable; il y a très peu de chance que, dans cette même couche de sol, une autre mesure donne une valeur inférieure à $10 \mathrm{kPa}$, mais, par contre, elle n'est que très peu informative, et il est difficile de faire des calculs ou de prendre des décisions sur cette base. Ce sont des valeurs numériques que l'on introduit dans des codes de calcul, parfois des distributions de probabilités, et c'est une valeur que l'on compare à une limite ou un index qui sert à prendre une décision. Que faire alors d'une information sous forme d'un intervalle ou d'un ensemble?

\section{5}

\section{L'utilisation des méthodes et les approches}

Les géotechniciens n'ont pas inventé de méthodes ou d'approches, ils se sont ingéniés, depuis plusieurs décennies, à adapter ce qui existe à leur problématique: Dans ce paragraphe, nous passons en revue un certain nombre de méthodes et d'approches et montrons, à travers des exemples d'application, quel est leur apport réel ou potentiel au domaine du sol urbain. Ces exemples n'ont aucune prétention à l'exhaustivité et proviennent essentiellement de travaux de recherche réalisés au LAMH à l'université d'Artois avec J. Al-Hajjar et C. Boulemia, puis au LGC à l'université BlaisePascal avec C. Bacconnet et R. Gourvès.

\section{1}

\section{Méthodes quantitatives}

\section{Lasial \\ La fiabilité}

La fiabilité d'un système est liée à son aptitude à remplir les fonctions pour lesquelles il a été conçu pendant une durée de temps déterminée.

La fiabilité des ouvrages en sol urbain relève de cette définition mais présente deux spécificités :

a) parce qu'ils sont cachés, il est difficile de suivre l'évolution temporelle de la dégradation d'une de leurs fonctions et, ainsi, de préciser à quel moment on atteint la limite de fonctionnement:

b) le comportement de l'ouvrage est à analyser dans le système complexe « sols/environnement/ouvrage »). Les causes de défaillance sont souvent difficiles à retrouver, car multiples et évolutives dans le temps, sous l'effet de sollicitations climatiques, et liées aux activités humaines, et dans l'espace.

Les hypothèses de base des méthodes fiabilistes sont que le système et les lois de comportement sont connues, que les paramètres de sol sont identifiés et quantifiés. Le résultat est un indicateur de la qualité d'une solution vis-à-vis de la fiabilité, Cet indicateur peut différer selon les modèles mécanofiabilistes retenus (déterministe : indice de Cornell, indice de Lind Hasofer (Hasofer A.M., Lind N.C., 1974), probabilité de ruine, possibilité de ruine...).

Il existe plusieurs niveaux de méthodes, allant des méthodes semi-probabilistes aux méthodes probabilistes, adoptant une approche semi-probabiliste, les eurocodes prennent en compte la variabilité des actions et leur possible concomitance, la variabilité des résistances. Il existe aussi des méthodes possibilistes basées sur la logique floue. Enfin, les méthodes basées sur les éléments finis stochastiques allient les apports des éléments finis et ceux des champs stochastiques. Une des limites de ces méthodes est qu'elles sont essentiellement utilisées en conception d'ouvrages nouveaux et mal adaptées à la requalification d'ouvrages existants.

Nous donnons ci-après quelques applications en fiabilité des fondations, de canalisations, d'ouvrages en terre: 
- Favre (1980), Biarez et al. (1981), Boissier (1982), Magnan (1982) ont travaillé sur l'approche probabiliste de la fiabilité des ouvrages enterrés :

- Boissier et al. (1995), Boissier et al. (1997) ont défini un index fiabiliste possibiliste pour l'évaluation des fondations, Gao (1996) l'a utilisé pour les structures ;

- Benmansour (1996) a travaillé sur l'évaluation probabiliste du comportement mécanique des conduites enterrées ;

- Auvinet (1994) a travaillé sur la prévision du comportement des barrages à partir de simulations stochastiques.

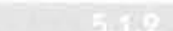

\section{La géostatistique}

Les travaux de (Matheron, 1970) ont grandement participé au développement de la géostatistique. Le point de départ de cette méthode est un système (sol, ouvrage...) connu à partir d'essais organisés et repérés dans l'espace; l'objectif est l'estimation d'une fonction des paramètres d'état de ce système dans des zones ponctuelles ou volumiques de l'espace. La méthode est bâtie sur la modélísation par champs aléatoires, dont les paramètres sont identifiés expérimentalement à partir des essais disponibles. Des résultats intermédiaires comme la forme des variogrammes ou la valeur des portées d'autocorrélation présentent des intérêts complémentaires sur la structure de variabilité spatiale ou la notion de VER, Les résultats sont l'estimation des grandeurs d'intérêt pour le problème posé, et la qualité de l'estimation traduite par la variance dont la valeur ainsi calculée est souvent réduite par rapport aux variances classiques (où l'on ne prend pas en compte la variabilité spatiale).

Cette approche est efficace sur des longs linéaires ou des grandes surfaces; en particulier elle a été appliquée sur les digues et sur les sols de tranchées urbaines, sur des surfaces importantes (remblais, plates-formes de bâtiments), son interprétation nécessitant un nombre de mesures important. Bacconnet (Bacconnet et al,, 1996) et Lepetit (Lepetit et al., 2002) ont utilisé cette technique pour l'étude de la résistance mécanique des digues, Lepetit (Lepetit et al., 1999) l'a utilisée pour étudier la variabilité spatiale du loess et Burlet (Burlet et al., 1999) celle de la neige, Boissier (Boissier et al., 1999) et Chaigneau (Chaigneau et al., 2000) pour modéliser la compacité de remblais de canalisations urbaines.

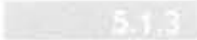

\section{La simulation}

La base de cette technique est un système dont on sait construire une maquette numérique, l'objectif étant d'avoir une représentation et une évaluation des différents états du système et de leur probabilité d'apparition, cette représentation pouvant être instantanée ou temporelle. La méthode est basée sur des techniques de simulation à partir de variables aléatoires, de champs stochastiques ou de champs possibilistes. En résultat on obtient une estimation des paramètres caractéristiques du comportement de l'ouvrage.

Ces méthodes de simulation ont été utilisées pour l'analyse du comportement d'un massif (prévision du risque d'avalanches, prévision du risque de fissuration des barrages...), d'un ouvrage (conduite d'assainissement). Boissier (1982) a utilisé cette technique pour l'évaluation probabiliste de la fiabilité des fondations superficielles, Auvinet (1994) pour celle des barrages en enrochement, Gaouar (1997) pour celle des digues et des remblais, Benmansour (1996) pour celle des canalisations enterrées, Burlet et al. (1999) pour celle des pentes neigeuses.

\section{L'analyse statistique}

L'analyse statistique part d'un système dont on analyse un ou des caractères à partir d'observations faites sur des échantillons, l'objectif étant d'obtenir une estimation des paramètres de la population à partir des observations faites sur l'échantillon. Les méthodes sont celles de l'analyse statistique incluant : les tests d'hypothèses, la théorie de l'estimation, les analyses en composantes principales, les analyses de corrélations, les analyses de variance et de covariance. Les résultats pratiques sont l'estimation de paramètres, la construction d'intervalles de confiance autour de moyennes, de proportions et de variances, l'analyse de régressions et de corrélations, l'adéquation de lois de probabilités à des distributions statistiques, la recherche de facteurs explicatifs principaux. Les applications concernant l'analyse d'actions, de sollicitations de résistance des matériaux à partir de statistiques sont classiques : d'autre applications concernent le traitement des signaux; récemment, Moussouteguy, et al. (2001) la utilisé pour l'analyse de paramètres de forage et Chaigneau (2001) pour l'analyse des signaux provenant d'essais pénétrométriques et pour l'analyse de répétatibilité d'essais. Courilleau (1997), puis Reche, et al. (2004) ont utilisé la théorie des lois de survie pour estimer, à partir de bases de données routières, l'état de dégradation des chaussées en tenant compte de l'entretien.

\section{Les plans d'expérience}

La méthode des plans d'expérience a pour objectif de minimiser le nombre d'expériences à faire pour étudier un phénomène tout en conservant une fiabilité suffisante aux résultats. Elle s'applique chaque fois qu'un phénomène peut être expliqué par plusieurs facteurs quantitatifs ou qualitatifs, que l'expérimentation soit numérique ou physique, le résultat pratique étant une proposition de l'organisation de l'expérimentation. Laffrechine (1999) a utilisé cette technique pour proposer des lois de survie des réseaux enterrés.

\section{2}

\section{Méthodes qualitatives}

\section{2 . \\ Analyse de compatibilité}

Cette approche est possible dés lors qu'un phénomène est décrit par plusieurs indicateurs souvent qualitatif's et que l'on souhaite construire une information unique sur ce phénomène. Elle emprunte de nombreux outils à la théorie des possibilités qui a été intro- 
duite par Zadeh (1965) puis diffusée en France par Dubois et Prades (Dubois, 1983) puis, parmi d'autres, par Bouchon-Meunier (1995). La méthode d'analyse de compatibilités est basée sur l'unification d'informations disparates, avec en particulier l'agrégation de sous-ensembles flous ; le résultat est, après défuzzyfication, l'obtention d'un couple cestimation de l'information, fiabilité de cette information » relativement au phénomène étudié.

Quelques applications d'analyse de compatibilité ont été réalisées en site urbain ; Semaan (1999) a analysé ainsi la compatibilité entre des informations provenant de plusieurs essais, Legendre (2003) entre celles provenant d'un sol et d'un outil de scarification ou de pénétration, Zeidan (2002) entre celles provenant d'un sol et d'un choix de tunnelier, et Alhaijar (1991) entre celles provenant d'un sol et d'un choix de fondations.

\section{2}

\section{Analyse des modes de défaillance}

Le point de départ est un système complexe dont on peut construire un schéma fonctionnel avec pour objectif la mise en évidence des défaillances potentielles de ce système et de leur criticité. Les méthodes sont issues de la sûreté de fonctionnement des systèmes industriels : méthode Hazop, des causes, des conséquences ; la plus utilisée actuellement en génie urbain étant la méthode AMDEC. Le résultat de la méthode AMDEC est une liste exhaustive de tous les scénarios de défaillance possible et un classement visà-vis de la criticité de ces scénarios. Cette méthode a été appliquée à l'analyse des modes de défaillance des barrages (Peyras, 2002), des réseaux (Bounader, 1998 ; Benmansour, 1996), des digues (Lepetit, 2002).

\section{$5 \times 9.9$}

\section{Analyse de sensibilité}

L'objectif de cette catégorie de méthodes est de permettre la caractérisation de la réponse d'un système complexe à la sensibilité de certains paramètres. La méthode est basée sur une description qualitative des phénomènes, de leur sens de variation, de leur gradient de variation, et éventuellement de l'accélération de cette variation. Cette description peut se faire à travers des variables bi ou multivaluées. Le résultat peut se mettre sous la forme d'une matrice de sensibilité du système à chaque facteur (ou combinaison de facteurs) selon leur intensité, leur évolution prévisible ou possible. Une application faite par Peyras (2001) concerne l'étude du vieillissement des barrages et de l'évolution des causes de défaillance.

\subsection{4}

\section{Le recueil d'expertise et le raisonnement expert}

Cette approche peut ètre utilisée lorsque on est en présence d'un problème complexe dont la résolution ne peut être explicitée analytiquement ; l'objectif est de comprendre ce phénomène et d'en expliquer les mécanismes à partir de dires d'expert ; la méthode consiste, en se basant sur différentes techniques (protocole, interviews, raisonnement par analogie...), à recueillir de l'expertise et à la transcrire sous forme logique essentiellement par l'écriture de règles de production (règles, métarègles...) ; le résultat prend souvent la forme de tables d'inférences ou de systèmes experts. E. Henry (1999) et Boissier (1999b) ont utilisé cette approche pour l'aide à la proposition d'une campagne de sol, Zeidan (2002) pour l'aide à la planification des tunneliers en site urbain, Karnib (2002) pour évaluer la sensibilité des zones urbaines aux dysfonctionnements des réseaux d'eaux pluviales.

\section{L'unification}

Cette approche est utilisable lorsqu'un même phénomène est décrit par plusieurs experts de façon parfois contradictoire ; l'objectif est d'avoir une vision unique prenant en compte cet ensemble de points de vue et présentant le maximum d'informativité et de fiabilité. Cette approche est basée sur une méthode développée par Shaffer (1976) ; le résultat est le triplet : « information-fiabilité-informativité n. Lair (1999) a utilisé cette approche pour évaluer la durabilité de produits du bâtiment et Semaan (1999) pour l'unification de résultats entre essais.

\section{6}

\section{Conclusion}

L'approche statistique heurte bien des idées acquises ; on oublie sans cesse la variabilité, on tient compte de différences dues au seul hasard, on conclut d'emblée de la liaison à la causalité, On peut se demander pourquoi ces erreurs sont si fréquentes. Les raisons sont multiples et s'enchevêtrent intimement : le calcul des probabilités a été inventé plus tard que beaucoup d'autres sciences, la statistique aussi car elle lui a été directement liée; nous sommes éduqués au lycée et formés à la vie dans l'idée de la certitude et de plus l'incertain nous fait peur. D'autre part, le sol urbain et ses ouvrages semblent tellement complexes que deux approches s'opposent constamment ; il y a ceux qui considérant que tout modèle serait illusoire essaient de l'oublier et de le noyer dans un poste incompressible d'aléas divers ; il y a ceux qui conscients de cette complexité construisent des modèles déterministes de plus en plus sophistiqués et avec toujours plus de paramètres pour reproduire une réalité qui leur échappera car elle sera toujours différente de celle du laboratoire et parce qu'ils n'auront pas les moyens d'alimenter ces modèles par des paramètres réalistes.

Les exemples présentés ici montrent qu'il y a place pour une troisième voie qui consiste d'abord et surtout à analyser et comprendre tout ce que, dans un projet, on peut mettre sous le terme générique d'imprécision , ensuite il faut choisir dans la panoplie des outils issus des théories des probabilités et des possibilités ceux qui sont le mieux adaptés à la modélisation, au traitement et aussi à l'exploitation des résultats dans le cadre opérationnel du génie urbain.

Il ne faut alors plus opposer information quantitative et information qualitative mais les utiliser concurremment afin d'optimiser, pour les données, les couples « informativité-fiabilité ). 
Alhajjar J., Boissier D., Boulemia J, - Building Substructural design as fuzzy decision model. 6th ICASP, Mexico. Mexique, juin 1991.

Auvinet G. - Modélisation stochastique de la rêpartition spatiale des propriétés des sols et ouvrages en terre. Journée nationale d'applications des statistiques et des probabilités en analyse des matériaux et des ouvrages, ENS Cachan. mars 1994, p. 159-171.

Bacconnet C., Boissier D., Gourves R. Aide au diagnostic des diques en terre par pénétrométrie. Colloque scientifique de I'AUGC, $\operatorname{COS}^{\prime} 96$, ClermontFerrand, 9-10 mai 1996

Alhaijar J., Boissier D., Boulemia J. - Buil ding substructural design as fuzzy decision model. 6th ICASP Mexico. Mexique, juin 1991.

Benmansour A. - Fiabilité des conduites enterrées. Thèse de l'institut national polytechnique de lorraine, 1996.

Biarez J., Favre J.-L., Lareal P., Boissier D. Probabilisme : caractérisation des sols, mesures de la sécurité, 10 e Congrés international de mécanique des sols. Stockhoim, 1981.

Boissier D. - Contribution à la prise en compte des interactions sol-fondations. bâti dans la conception des bátiments. Approche probabiliste de la sécurité. Thèse de docteur ès sciences, Université de Lyon I, INSA de Lyon, 2 juillet 1982.

Boissier D. Alhaijar J., Mammeri H. - Fiabilité et possibilité en mécanique des sols: application à l'infrastructure des bâtiments. $12^{e}$ congrès de mécanique. Strasbourg. 4-8 septembre 1995.

Boissier D. Alhailiar J. - Probabilistic and fuzzy approaches for foundations reliability, ICOSSAR'97, Kyoto, Japan nov. 24-28th 1997.

Boissier D., Bacconnet C., Morales E. Modélisation de la variabilité spatiale des tranchées d'assainissement; application au collecteur de La Pardieu. Journées scientifiques sols urbains, GIS sols urbains, université de Bordeaux I, 21-22 octobre 1999

Boissier D., Henry E., Boulemia C. - Aide à la conception de fondations de bâtiments: un modèle d'expertise. Revuc française de génie civil, Hermès, vol. 3. $\mathrm{n}^{\circ}$ 5, septembre 1999 .

Bouchon-Meunier B. - La logique floue et ses applications. Addison Wesley. Amsterdam, 1995, p. 84-92.

Bounader E. - Conduite du diagnostic et évaluation des collecteurs des infrastructures urbaines de l'assainissement. Thèse de l'INSA de Lyon, 1998.
Burlet J.-L., Bacconnet C., Boissier D. Gourves R. - Modeling of the snow cover variability with a gaussian stochastic field. ICASP 8, Sydney. R.E. Melchers et M.G. Stewart (eds) Balkema, 1999, p. 869-875.

Chaigneau L., Boissier D., Bacconnet C. Gourves R. - Mesure in situ et estima. tion de la variabilité des sols. Journées de l'école doctorale SPI, Clermont-Ferrand, mars 2000

Chaigneau L. - Caractérisation des milleux granulaires à l'aide d'un pénétromètre. Thèse de l'Université Blaise-Pascal, Clermont-Ferrand, 2001

Courilleau E. - Analyse statistique de données routières appliquée au développement de modeles de gestion de l'entretien. Thèse de l'Université BlaisePascal, Clermont-Ferrand, 1997.

Dubois D., Prade H. - Ranking fuzzy numbers in the sitting of possibility theory. Information sciences 30, Elsevier science publishing co., inc., New York, 1983, p. 183-224.

Favre J.-L. - Milieu continu et milieu discontinu : mesure statistique indirecte des paramètres rhéologiques et approche probabiliste de la sécurité. Thèse de doctorat es sciences, Université Paris VI, 1980.

Gao L. - Modèles probabilistes et possibi. listes pour la prise en compte de l'incertain dans la sécurité des structures. Thèse de I'ENPC Paris, 1996

Gaouar M. - Approche fiabiliste de la stabilité des barrages en terre par simulation de champs aléatoires. Thèse de IUniversité Blaise-Pascal, Clermont. Ferrand, 1997.

Hasofer A.M. Lind N.C. - Exact and second invariant second moment code format. Engineering mechanics division, ASCE 100, 1974

Henry E., Boissier D., Boulemia C. - Une modélisation orientée objet addaptée aux différents contextes de conception des fondations de bâtiments. Arnales du batiment et des travaux publics 4 . septembre-octobre 1999, p. 37-46.

Karnib A. Alhaijar J. Boissier D. - An expert system to evaluate the sensitivity of urban areas to the functioning failure of storm drainage networks. Urban Water 4, 2002, p. 43-51

Laffrechine K. - Base de donnees urbaine pour la gestion des réseaux d'assainissement non visitables. Thèse de l'université de Bordeaux 1. CDGA, 1999

Lair J., Le teno J.F. Boissier D. - An approach for durability assessment of building systems. International conference on applied statistics, ICASP 8,
Sydney, R.E. Melchers et M.G. Stewart (eds), Balkema, 1999, p. 285-290.

Legendre A - Reconnaissance des sols de surface ; application à la scarification des sols. Thèse de l'université BlaisePascal, Clermont-Ferrand, 2003.

Lepetit L., Bacconnet C., Boissier D. Gourves R. - Geostatistical study of the chinese loess, ICASP 8. Sydney, R.E. Melchers et M.G. Stewart (eds), Balkema, 1999, p. 491-498

Lepetit L., Bacconnet C., Boissier D. Gourves R. - Etude d’une méthode de diagnostic des digues. Annales du bätiment et des travaux publics 2, avrí 2002, p. 67-73.

Magnan J.-P - Les méthodes statistiques et probabilistes en mécanique des sols. Presses des ponts et chaussées, Paris, décembre 1982

Matheron G. - La théorie des variables régionalisées et ses applications. École des Mines de Paris, 1970

Moussouteguy N., Breysse D., Chassagne P. - Dimimution des incertitudes géotechniques par une meilleure reconnaissance de l'hétérogénéité du sol : utilisation combinée des diagra. phies instantanées et des essais pressiométriques. INFIAB 3, Université de Bordeaux I, 2001.

Peyras L., Royet P., Boissier D., Vergne A. Évaluation de la criticité du vieillissement des barrages par la modélisation fonctionnelle. $1^{\mathrm{N}}$ conférence internationale Albert Caquot. Presses des Ponts et chaussées, Paris, 2001.

Peyras L Royet P. Boissier D. Vergne A Diagnostic des dégradations des barrages : développement d'une méthodologie basée sur la modélisation fonctionnelle. Annales du bâtiment et des travaux publics 1, février 2002, p. 59-64.

Rèche M., Vergne A., Lepert Ph, - Impact of maintenance treatment on pavement cracking models. Fifth RILEN International Conference Cracking Pavement, Limoges, 5-8 mai 2004

Semaan I., Boissier D., Boulemia C. - Possibilistic modelling of data in a soil campaign. 1CASP 8. Sydney, R.E. Mel. chers et M.G. Stewart (eds), Balkema, 1999. p. 1115-1122.

Shafer G. - A mathematical theory of evidence. Princeton University Press. 1976, 297 p

Zadeh L.A. - Fuzzy sets, information and control, 8, 1965, p. 338-353.

Zeidan A.S. - Démarche d'estimation des délais de réalisation d'un tunnel en site urbain. Thèse de IUniversité de Marne. la-Vallée, Champs-sur-Marne, 2002. 